\title{
Glass transition dynamics and structural relaxation of PLLA studied by DSC: Influence of crystallinity
}

\author{
J.F. Mano ${ }^{\mathrm{a}, \mathrm{b}, *}$, J.L. Gómez Ribelles ${ }^{\mathrm{c}}$, N.M. Alves ${ }^{\mathrm{a}, \mathrm{b}}$, M. Salmerón Sanchez ${ }^{\mathrm{c}}$

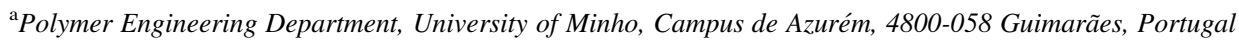 \\ b3B's Research Group-Biomaterials, Biodegradables and Biomimetics, University of Minho, Campus de Gualtar, 4710-057 Braga, Portugal \\ ${ }^{\mathrm{c}}$ Center for Biomaterials, Universidad Politécnica de Valencia, P.O. Box 22012, E-46071 Valencia, Spain
}

Received 9 December 2004; received in revised form 28 May 2005; accepted 10 June 2005

Available online 20 July 2005

\begin{abstract}
Poly(L-lactic acid), PLLA, in amorphous and semi-crystalline forms were studied by DSC, in order to investigate both molecular dynamics and structural relaxation features, and to understand the influence of the crystalline confinement on the segmental mobility of the intraspherulitic amorphous phase. Experimental data were generated after submitting the materials to different thermal histories below $T_{\mathrm{g}}$ and were treated with a model based on the configurational entropy concept, allowing to extract a series of physical-meaningful parameters and to obtain the temperature dependence of the relaxation times. The main features of the relaxation process in the glassy state (activation energy of the glassy state and $x$ ) and the fragility index were found to be apparently insensitive to crystallinity. Significant differences between the two materials were detected in both the position of the glass transition temperature and the width of the distribution of relaxation times. In the framework of the Adam and Gibbs theory, it is suggested that for the semi-cristalline PLLA the mobility of the amorphous chains is more or less restricted, depending on their distance to the rigid lamellar walls or on the intra-lamellar thickness; this will imply that their conformational motions will take place at different temperatures, typically above the glass transition temperature of the (unconfined) bulk amorphous phase.
\end{abstract}

(C) 2005 Published by Elsevier Ltd.

Keywords: Semi-crystalline polyesters; Polylactides; Glass transition

\section{Introduction}

Poly(L-lactic acid), PLLA, is a well known biodegradable and biocompatible polymer. Such properties, allied with the processability and good mechanical properties make this polymer adequate to be used in many biomedical applications [1-3] such as wound closure, prosthetic implants, controlled-released systems and three-dimensional scaffolds. PLLA exhibits low crystallisation kinetics [4-6], being easy to prepare this material with a wide range of degrees of crystallinity. This parameter will determine many physical properties (including mechanical behaviour) and may affect the biological response (e.g. cell growth and

\footnotetext{
* Corresponding author. Address: Polymer Engineering Department, University of Minho, Campus de Azurém, 4800-058 Guimarães, Portugal. Tel.: + 351 253510320; fax: + 351253510339 .

E-mail address: jmano@dep.uminho.pt (J.F. Mano).
}

0032-3861/\$ - see front matter (C) 2005 Published by Elsevier Ltd. doi:10.1016/j.polymer.2005.06.096 proliferation in culture). The morphology and particularly the degree of crystallinity will influence also the hydrolytic degradation kinetics in a clinical situation. It has been found that the rate of hydrolytic or enzymatic degradability of polylactides is strongly affected by the degree of crystallinity and lamellar organisation [7-10].

Depending on annealing conditions different morphologies and crystallinity may be attained, having a considerable impact on the final materials' properties [11]. However, even in the most favourable cases, it is hard to achieve crystallinity degree in PLLA above 60\% [11]. Therefore, the amorphous fraction should play an important role on the final properties of this polymer. In biomedical applications this is especially relevant as the glass transition temperature of PLLA is about $60-65^{\circ} \mathrm{C}$, i.e. not far above physiological temperature. Therefore, the amorphous fraction of any PLA-based implanted material will be in the glassy state. As $T_{\mathrm{g}}$ is not very distant from $37^{\circ} \mathrm{C}$, the system will slowly approach the equilibrium at this temperature. This structural relaxation phenomenon, or physical ageing, is a kinetic 
phenomenon that results from the non-equilibrium characteristic of the glassy state. It manifests as, for example, an apparent shift of the creep response to longer times or a reduction in the specific volume $V$ or enthalpy $H$. So, physical ageing may contribute for the change of the physical properties of PLLA with time at the moment that it is implanted, besides the pure chemical effect of the degradation by hydrolysis of the chains. Some issues related to the structural relaxation in PLLA or PDLLA were addressed before [12,13]. However, a more deep and quantitative inspection of structural relaxation and general glass transition dynamics is still necessary, that should include the determination of the distribution and temperature dependence of the characteristic times for segmental mobility and the effect of crystallinity. This relationship is particularly important as it has been shown, from differential scanning calorimetry (DSC) measurements, that the crystalline regions constrain the amorphous phase and influence its conformational motions [14-17]. The influence of crystallinity on the glass transition dynamics has been widely investigated in poly(ethylene terephthalate), PET [14-20], but this effect has been scarcely studied in poly(lactic acid)-based systems, and probed mainly by dielectric relaxation spectroscopy [21,22]. PLLA is a polyester, such as PET, but the lamellar morphology is quite different; for example, PLLA exhibits higher long spacing than PET. Therefore, the study of this system may bring new insights on the general discussion on the effect of the crystalline phase on the segmental dynamics, a fundamental problem that is far to be fully understood.

In this work, the glass transition features of a PLLA material are investigated by means of DSC. Scans obtained after distinct thermal treatments, including stages at different annealing temperatures below $T_{\mathrm{g}}$ and times, or coolings at different rates, are treated with a phenomenological model. This allows to extract a series of physical parameters related to the temperature and structure dependence of the characteristic time for the conformational rearrangements of the polymer segments that are responsible for the relaxation of enthalpy taking place within the mobile amorphous phase. This procedure will be applied in two distinct forms of PLLA: Nearly amorphous and with a high degree of crystallinity (fully spherulitic morphology). This will allow to characterise the glass transition dynamics in PLLA when, on one hand, the amorphous chains are free of constrains imposed by the crystalline region and, on the other hand, the amorphous chains are confined within the crystalline lamellae.

\section{Model based on configurational entropy}

The evolution of the configurational entropy during a thermal history that consists of a series of temperature jumps from $T_{i-1}$ to $T_{i}$ at time instants $t_{i}$, followed by isothermal stages is given by [23-27]:

$$
\begin{aligned}
S_{\mathrm{c}}(t)= & S_{\mathrm{c}}^{\lim }(T(t))-\sum_{i=1}^{n}\left(\int_{T_{i-1}}^{T_{i}} \frac{\Delta C_{\mathrm{p}}^{\lim }(T)}{T} \mathrm{~d} T\right) \\
& \times \exp \left(-\left(\xi-\xi_{i-1}\right)^{\beta}\right)
\end{aligned}
$$

where $\beta$ is the Kohlrausch-Williams-Watts, KWW, parameter, that takes into account the non-exponential behaviour of the segmental mobility:

$\phi(\xi)=\exp \left(-\xi^{\beta}\right)$

where $\xi$ is the reduced time:

$\xi=\int_{0}^{t} \frac{\mathrm{d} t^{\prime}}{\tau\left(t^{\prime}\right)}$

The function $\tau(t)$ is determined implicitly by the dependence of $\tau$ on $T$ and $S_{\mathrm{c}}$ during the thermal history, a dependence which is assumed to obey the Adam and Gibbs equation [28] extended to non-equilibrium states:

$\tau\left(T, S_{\mathrm{c}}\right)=A \exp \left(\frac{B}{T S_{\mathrm{c}}(t, T)}\right)$

$S_{\mathrm{c}}^{\lim }(T)$ represents the value of the configurational entropy attained in the physical ageing process at infinite time, and $\Delta C_{\mathrm{p}}^{\lim }(T)$ is defined through

$S_{\mathrm{c}}^{\lim }(T)=S_{\mathrm{c}}^{\mathrm{eq}}\left(T^{*}\right)+\int_{T^{*}}^{T} \frac{\Delta C_{\mathrm{p}}^{\lim }(T)}{T} \mathrm{~d} T$

with $T^{*}$ a temperature high enough above the glass transition and

$S_{\mathrm{c}}^{\mathrm{eq}}(T)=\int_{T_{2}}^{T} \frac{\Delta C_{\mathrm{p}}(T)}{T} \mathrm{~d} T$

where $\Delta C_{\mathrm{p}}(T)$ is the configurational heat capacity, here taken as the difference between the heat capacities of the liquid and the glass, $\Delta C_{\mathrm{p}}(T)=C_{\mathrm{pl}}(T)-C_{\mathrm{pg}}(T)$ (a linear dependence of $\Delta C_{\mathrm{p}}(T)$ with temperature has been assumed in this work) and $T_{2}$ is the Gibbs-DiMarzio temperature at which the configurational entropy in the equilibrium liquid would vanish.

Phenomenological models of structural relaxation usually assume that the state attained at infinite time in the structural relaxation process at a temperature $T_{\mathrm{a}}$ can be identified with the extrapolation to $T_{\mathrm{a}}$ of the equilibrium line experimentally determined at temperatures above $T_{\mathrm{g}}$. When the models are based on the fictive temperature $\left(T_{\mathrm{f}}\right)$ concept this is simply a result of the identification of the limit of $T_{\mathrm{f}}$ at infinite time with $T$. In the context of the model used in this work this means that:

$S_{\mathrm{c}}^{\lim }(T)=S_{\mathrm{c}}^{\mathrm{eq}}(T)$

However, it has been shown that the agreement between the model simulation and the experiments is highly improved when the model includes an assumption leading to values of $S_{\mathrm{c}}^{\mathrm{lim}}(T)$ significantly higher than those of $S_{\mathrm{c}}^{\mathrm{eq}}(T)$ [23-27]. The definition of the curve $S_{\mathrm{c}}^{\lim }(T)$ introduces new 
adjustable parameters in the model. The shape shown in Fig. 1 has been chosen since it needs only one additional parameter $\delta$, defined as shown in the figure. Therefore, the model has five adjustable parameters: $\delta$, the pre-exponential constant $A$ and the parameter $B$ of the Adam-Gibbs equation, the Gibbs-DiMarzio temperature $T_{2}$ and the exponent $\beta$ of the KWW equation.

\section{Materials and methods}

The polymerization of PLLA was carried out as described elsewhere [29]. Briefly, a glass polymerization reactor equipped with a nitrogen flow-through inlet and a vacuum connection, was placed in a temperature-controlled bath containing silicone oil. Polymerization was performed under nitrogen atmosphere at the temperature range of 100 $150{ }^{\circ} \mathrm{C}$ for $12-48 \mathrm{~h}$. In order to remove residual monomers, chloroform and methanol were used as the solvent and precipitant, respectively. The molecular weights of the polymer, $M_{\mathrm{n}}$ and $M_{\mathrm{w}}$, were 58,000 and 132,000, respectively, evaluated from gel permeation chromatography (Shimadzu, LC 10A, Japan) using polystyrene as standard and chloroform as solvent. The melting temperature of the obtained material is $169^{\circ} \mathrm{C}$ (onset temperature, obtained by DSC at $20^{\circ} \mathrm{C} \min ^{-1}$ ).

The thermal properties were investigated in a PerkinElmer Pyris equipment, using the conventional calibration methods with high pure standards. Two kinds of PLLA sample were analysed: A nearly-amorphous material, NA-PLLA, and a semi-crystalline PLLA, SC-PLLA.

NA-PLLA was obtained by cooling the material in the DSC pan at $40{ }^{\circ} \mathrm{C} \mathrm{min}{ }^{-1}$ from $190{ }^{\circ} \mathrm{C}$ down to $20^{\circ} \mathrm{C}$. SC-PLLA was obtained by cooling the polymer at $1{ }^{\circ} \mathrm{C} \mathrm{min}^{-1}$ from 190 to $20^{\circ} \mathrm{C}$. In Section 4 are shown the DSC results

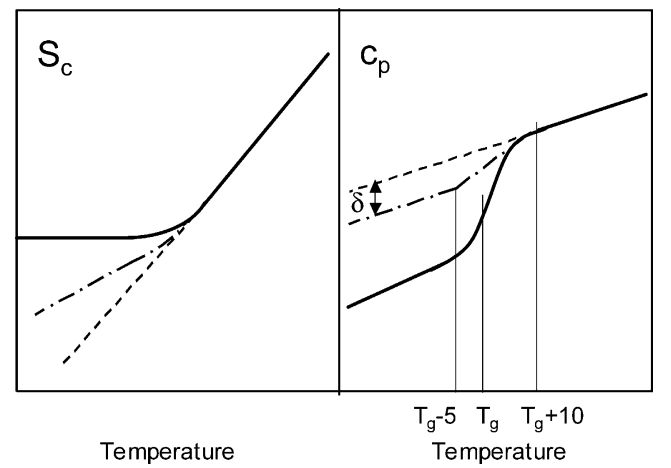

Fig. 1. Left, sketch of the configurational entropy corresponding to the liquid state (dashed line), to an experimental cooling scan at a finite cooling rate (solid line), and to the hypothetical line of the limit states of the structural relaxation process (dashed-dotted line). Right, $C_{\mathrm{p}}(T)$ lines corresponding to the three cases described in the left graphics: The dashed line corresponds to the liquid state $C_{\mathrm{pl}}(T)$, the solid line corresponds to an experimental cooling scan, and the dashed-dotted line corresponds to the specific heat capacity in the limit states of the structural relaxation process: $C_{\mathrm{p}}^{\lim }(T)$. for samples previously cooled at different rates from the melt, and the reasons for choosing these particular treatments for obtaining both NA-PLLA and SC-PLLA are explained.

When a phenomenological model such as the one used in this work is applied in structural relaxation studies, it is important to subject the sample to several and distinct thermal histories in order to obtain parameters characteristic of the material, i.e. independent of thermal history. Such procedure was widely discussed previously [23-27]. So, for both NA-PLLA and SC-PLLA the following thermal histories were used:

(i) Cooling from the equilibrium state (i.e. above $T_{\mathrm{g}}$ ) at different rates.

(ii) Cooling at $40{ }^{\circ} \mathrm{C} \mathrm{min}^{-1}$ from the equilibrium state down to different ageing temperatures $\left(T_{\mathrm{a}}<T_{\mathrm{g}}\right)$. The sample was kept at $T_{\mathrm{a}}$ during different ageing times $\left(t_{\mathrm{a}}\right)$, prior to the final conventional heating DSC scans that were carried out at $20{ }^{\circ} \mathrm{C} \mathrm{min}^{-1}$ from 20 to $90{ }^{\circ} \mathrm{C}$.

Regarding the thermal treatments referred in (ii), and for SC-PLLA, the initial fast cooling at $40{ }^{\circ} \mathrm{C} \mathrm{min}-1$ started always at $90{ }^{\circ} \mathrm{C}$ and it is not expected any change in the crystallinity due to the successive DSC scans. Nevertheless, for NA-PLLA significant crystallization could take place due to successive 'annealings' at $90^{\circ} \mathrm{C}$. Thus, in this case the thermal treatment started in the melt and the sample was first cooled down to the ageing temperature $T_{\mathrm{a}}$ at $40{ }^{\circ} \mathrm{C} \mathrm{min}{ }^{-1}$. For the particular case of the thermal history consisting in the cooling through the glass transition at $0.5^{\circ} \mathrm{C} \mathrm{min}{ }^{-1}$, and also in order to avoid crystallisation, the NA-PLLA sample was cooled from the melt to $70{ }^{\circ} \mathrm{C}$ at $40{ }^{\circ} \mathrm{C} \mathrm{min}{ }^{-1}$ and then from 70 to $20{ }^{\circ} \mathrm{C}$ at $0.5^{\circ} \mathrm{C} \mathrm{min}{ }^{-1}$.

The characterisation of the crystallisation features was carried out using TMDSC and conventional DSC, in both isothermal and scanning modes, and published elsewhere [30]. Crystallization experiments at different cooling rates were also performed under cross-polarised optical microscopy and presented in a previous work [31].

\section{Results}

There are different ways to induce and control crystallinity in PLLA. A final microstructure may result from an isothermal annealing by quenching the liquid (or heating the amorphous glass below $T_{\mathrm{g}}$ ) to a given temperature between $T_{\mathrm{g}}$ and $T_{\mathrm{m}}$, during a particular time. In this case, the temperature should be carefully calibrated if one pretends to reproduce the same thermal treatment. Alternatively, the cooling from the liquid state at different rates will also lead to materials with different degrees of crystallinity. The final morphology will be essentially dependent on the cooling rate, and will not be very sensitive either to the temperature 
calibration of the apparatus nor on the sample's thickness. Fig. 2 shows a series of DSC heating curves, in the same material that was subjected to a previous cooling from the melt down to room temperature at different rates.

A detailed analysis relating the cooling rate of the thermal treatment and the final microstructure for the same PLLA is described elsewhere [31]. It was found that for the highest cooling rates (above $5{ }^{\circ} \mathrm{C} \mathrm{min}{ }^{-1}$ ) the material is essentially amorphous [31]. This was revealed by the nonexistence of a diffraction pattern in X-ray scattering experiments (WAXS and SAXS) conducted on a material previously cooled above $5{ }^{\circ} \mathrm{C} \min ^{-1}$. This means that a small quantity of small crystallites may still exist in this material, although they are not able to diffract X-rays.

Moreover, from the data presented in Fig. 2, the integration of the DSC trace covering both crystallisation and melting peaks for a sample prepared as mentioned above, gives a negligible enthalpy, characteristic of a nearly amorphous material.

Taking into account the previously referred work [31] and the results of Fig. 2, we will use the material cooled at $40{ }^{\circ} \mathrm{C} \min ^{-1}$ as the nearly-amorphous PLLA (NA-PLLA) in the structural relaxation studies. On the other hand, the DSC trace of the sample previously cooled at $1{ }^{\circ} \mathrm{C} \mathrm{min}^{-1}$ does not exhibit the cold-crystallisation peak providing an indication that a deep crystallisation process took place during the previous cooling (Fig. 2). The morphology, as seen by cross polarised optical microscopy, shows a fully spherulitic structure for a sample prepared in these conditions [31]. This semi-crystalline sample, designated as SC-PLLA, will be used in the structural relaxation studies to investigate the case of an amorphous phase confined within the crystalline structure. The crystalline fraction, $x_{\mathrm{c}}$, can be estimated from DSC results by using the relation $x_{\mathrm{c}}=\Delta H_{\mathrm{m}} / \Delta H_{\mathrm{m}}^{0}$, where $\Delta H_{\mathrm{m}}$ is the measured enthalpy of fusion of the sample, $\Delta H_{\mathrm{m}}^{0}$ is the enthalpy of fusion of the completely crystalline material (i.e. corresponding to an infinite thickness crystal). Assuming values of $81.3-93 \mathrm{Jg}^{-1}$ for the enthalpy of fusion of the infinite thickness crystal

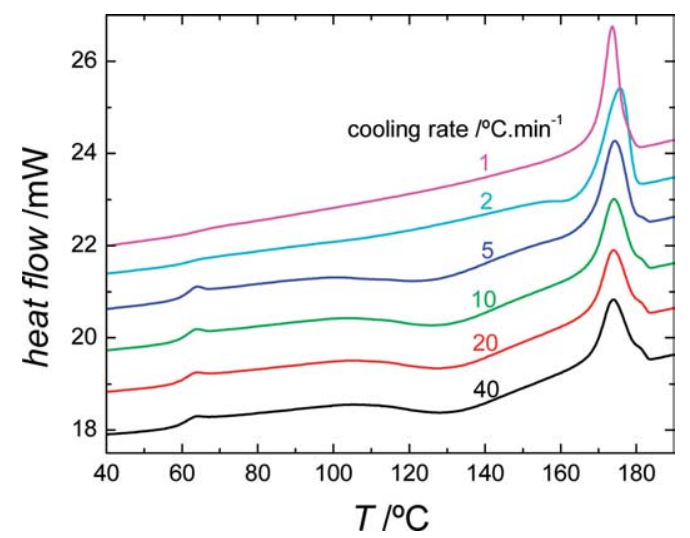

Fig. 2. DSC heating scans at $20^{\circ} \mathrm{C} \mathrm{min}-1$, from 30 to $200{ }^{\circ} \mathrm{C}$, of the same PLLA sample after cooling from the melt at different rates (indicated on each curve).
[32], the crystalline fraction of this material, $x_{\mathrm{c}}$, is between 0.24 and 0.28 .

Figs. 3 and 4 show the DSC scans at $20^{\circ} \mathrm{C} \mathrm{min}^{-1}$ after different thermal histories for NA-PLLA and SC-PLLA, respectively (symbols). From the analysis of the ensemble of these results it may be seen that glass transition temperature is higher for the crystalline material. This happens because the mobility of the chain segments, which are necessary to promote the recovery of the enthalpy, decreases with crystallinity. This hindered mobility is the result of the existence of a geometrical constraint imposed by the crystallites to the conformational mobility within the amorphous fraction. Moreover, the glass transition range is broader for SC-PLLA and the intensity of the change (both the step in the heat flow and intensity of the endothermic peaks) decreases from NA-PLLA to SC-PLLA, reflecting the decrease of the amorphous phase content. The ratio of $\Delta C_{\mathrm{p}}$ increment at the glass transition temperature in SC-PLLA to that of NA-PLLA is 0.17 , much smaller than what would be expected from the crystallinity of the sample, what means that a significant fraction of the chain segments that are out of the lamellae do not have enough conformational mobility to participate in the glass transition. The existence of such amount of material that is not involved in the glass transition process suggests that the amorphous material near the crystallites differs from the normal polymer melt. Local equilibrium between the crystallites and the adjacent amorphous layers has been suggested in literature as an explanation of the so-called surface crystallization and melting phenomenon as evidenced, e.g. by SAXS experiments on polyethylene [33], modulated calorimetry [34,35] and Raman spectroscopy [36]. These experiments show that a change in temperature results in maintaining the thickness of the transition layer between the crystallites and the melt, even though there is a continuous shift of the interface as crystallinity changes.

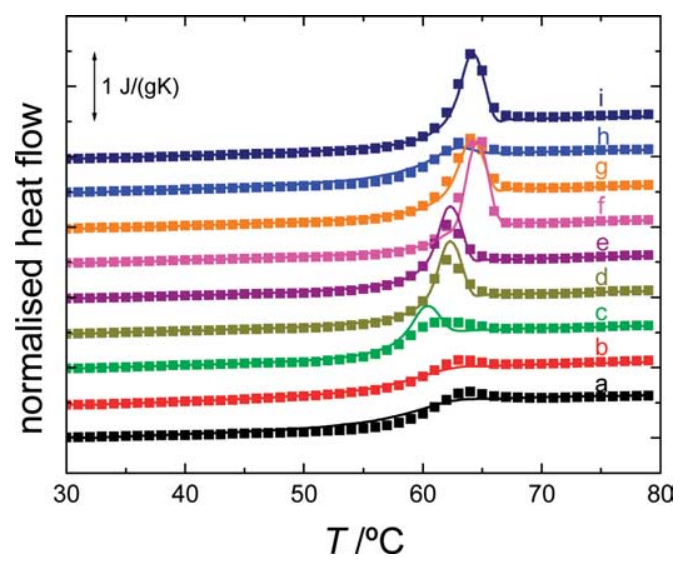

Fig. 3. DSC heating scans at $20^{\circ} \mathrm{C} \mathrm{min}^{-1}$ obtained with the NA-PLLA sample, after different thermal histories (points): Reference scan with a quenched sample (a) $180 \mathrm{~min}$ ageing at different temperatures (b) $25^{\circ} \mathrm{C}$, (c) $35^{\circ} \mathrm{C}$, (d) $40{ }^{\circ} \mathrm{C}$, (e) $45^{\circ} \mathrm{C}$, (f) $50^{\circ} \mathrm{C}$, (g) $55^{\circ} \mathrm{C}$ and (h) $60^{\circ} \mathrm{C}$ and cooling at (i) $0.5^{\circ} \mathrm{C} \mathrm{min}{ }^{-1}$. The lines are the corresponding fittings according to the used model (fitting parameters are in Table 1). 


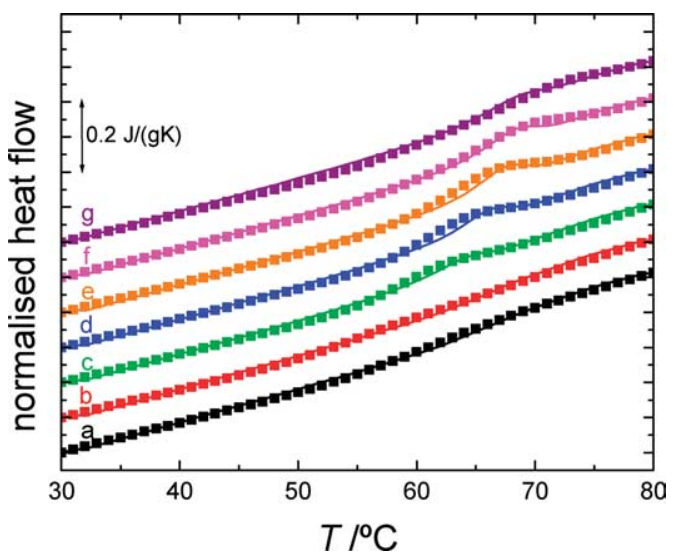

Fig. 4. DSC heating scans at $20{ }^{\circ} \mathrm{C} \mathrm{min}{ }^{-1}$ obtained with the SC sample, after different thermal histories (points); from the bottom: Reference scan with quenched sample (a) and 180 min ageing at different temperatures (b) $30{ }^{\circ} \mathrm{C}$, (c) $40{ }^{\circ} \mathrm{C}$, (d) $45^{\circ} \mathrm{C}$, (e) $50{ }^{\circ} \mathrm{C}$, (f) $55^{\circ} \mathrm{C}$ and (g) $60{ }^{\circ} \mathrm{C}$. The lines are the corresponding fittings according to the used model (fitting parameters are in Table 1).

The chain structure of these layers consists of loops, which are fixed with their ends in the crystallites and trapped entanglements in a concentration higher than that in the normal polymer melt, as a consequence the chemical potential of these units differs from that in the bulk melt. The high surface to volume ratio of the crystallites accounts for such a high amount of material that not being able to crystallise cannot participate in the glass transition process.

Especially for PLLA, it is visible that, for the same ageing time (180 $\mathrm{min}$ ), and starting from the lowest ageing temperatures, $T_{\mathrm{a}}$, there is an increase of the endothermic peak height as $T_{\mathrm{a}}$ increases, because the approach to equilibrium is faster as $T_{\mathrm{a}}$ increases towards $T_{\mathrm{g}}$. On the other hand, for a sufficiently high $T_{\mathrm{a}}$, close to $T_{\mathrm{g}}$, the rate of the structural relaxation process is high, but the variation of enthalpy produced is small because the material is already close to equilibrium at the beginning of the structural relaxation process. A further increase of $T_{\mathrm{a}}$ would lead to a value at which there is no difference between the scan measured on the aged sample and the reference scan.

Different sets of the heating scans for NA-PLLA and SC-PLLA, chosen among the available data (Figs. 3 and 4), were fitted with the phenomenological model described in Section 2. In this case, we consider a linear dependence of $\Delta C_{\mathrm{p}}$ with temperature [37] and used it for the model calculations. The simulations were conducted for all the samples under the assumption $S_{\mathrm{c}}^{\lim }(T)>S_{\mathrm{c}}^{\mathrm{eq}}(T)$, i.e. assuming that the equilibrium state will not be reached. It was previously found that the fitting parameters of the model, $\ln A, B, \beta, T_{2}$ and $\delta$ are not statistically independent, but rather different combinations of sets of parameter could lead to similar representations of the temperature dependence of the relaxation times [24-27]. Then, the fitting procedure requires the fixation of a given parameter, usually $B$, from 500 to $1000 \mathrm{Jg}^{-1}$, and the four other parameters are determined using a least-square routine. After different attempts, the best value of $B$ is judged according to the realistic values of $A$ and $T_{\mathrm{g}}-T_{2}$. However, in this work, $A$ was fixed as $10^{-14} \mathrm{~s}$ for both NA-PLLA and SC-PLLA. As commented by Angell [38], $A$ is seen as a microscopic quantity, with values of this order of magnitude, related to the frequency of attempts to cross some barrier opposing the rearrangement of particles involved in the relaxation $[28,39]$, or the time a molecule needs to move into some free space $[40,41]$. More details about the fitting procedure can be found elsewhere [23-27].

The fitted values of $B, \beta, T_{2}$ and $\delta$ are shown in Table 1 , for both materials. The uncertainty of each parameter was estimated according to the procedure described elsewhere [42]. It is assumed that these parameters are materials' parameters, independent from the thermal history. The reproduction of the DSC curves using these results are shown in Figs. 3 and 4. In general it can be said that the fits are satisfactory for the two samples.

\section{Discussion}

As pointed out before, the DSC results suggest a broadening of the glass transition when PLLA is crystallised. This can be due to different reasons, as commented in a previous work [17]: (i) A decrease in the apparent activation energy around $T_{\mathrm{g}}$; (ii) an increase of composition heterogeneity, i.e. a broadening of the distribution of distinct phases (nanometric amorphous regions with different sizes) with different $T_{\mathrm{g}} \mathrm{s}$ or (iii) an intrinsic broadening of the relaxation times distribution. At least, the last hypothesis could be viable since, on one hand, for SC-PLLA the fitting quality was acceptable, which does not occur in very heterogeneous systems; on the other hand, a significant difference in the $\mathrm{KWW}$ parameter $\beta$ values (Eq. (2)) is found, which is an indication of differences in the distribution of characteristic times. For NA-PLLA $\beta=$ $0.40 \pm 0.006$ which is very close to the $\mathrm{KWW}$ stretch parameters found in a series of amorphous poly(D,L-lactic acid) with different molecular weights, where $\beta=0.41 \pm$ 0.01 for all samples [43]. For SC-PLLA we find $\beta=0.35 \pm$ 0.006 , indicating a more varied and constrained mobility of the polymeric segments within the crystalline lamellae, with respect to the bulky amorphous phase. In the semicrystalline material the close interaction with crystallites enhances the possibility of finding amorphous regions with different mobility. A decrease of $\beta$ was also detected in PET, when crystallisation was induced in the system [17].

Table 1 shows that no significant differences exist in the diverging temperature $T_{2}$ of the two studied materials. The differences found in $B$ mainly reflect the differences in $\Delta C_{\mathrm{p}}$ between NA-PLLA and SC-PLLA. It was found that $\Delta C_{\mathrm{p}}(\mathrm{NA}-\mathrm{PLLA}) / \Delta C_{\mathrm{p}}(\mathrm{SC}-\mathrm{PLLA})=5.9$, similar to $B(\mathrm{NA}-$ PLLA $) / B($ SC-PLLA $)=4.9 \pm 0.5$. The correlation between $B$ and $\Delta C_{\mathrm{p}}$ is due to the form of the Adam-Gibbs equation 
Table 1

Model parameters describing the glass transition dynamics of NA-PLLA and SC-PLLA, using the experimental data in Figs. 3 and 4

\begin{tabular}{lllll}
\hline & $\ln A(\mathrm{~s})$ & $B\left(\mathrm{Jg}^{-1}\right)$ & $\beta$ & $T_{2}\left({ }^{\circ} \mathrm{C}\right)$ \\
\hline NA-PLLA & -32.24 & $556 \pm 2$ & $0.40 \pm 0.006$ & $25.4 \pm 1$ \\
SC-PLLA & -32.24 & $113 \pm 6$ & $0.35 \pm 0.006$ & $0.055 \pm 0.01$ \\
\hline
\end{tabular}

[Eq. (4)] and the dependence of the configurational entropy with $\Delta C_{\mathrm{p}}$ (Eqs. (5) and (6)).

The ratio $\delta / \Delta C_{\mathrm{p}}$ is small in NA-PLLA $\left(\delta / \Delta C_{\mathrm{p}}=0.13\right)$, indicating that the limit state for structural relaxation coincides almost with the extrapolated equilibrium state. A reason for this finding may be related to the quite flexible nature of the PLLA chains. Below $T_{\mathrm{g}}$ the topological constraints, which obstruct the evolution towards the equilibrium state, may be easier to surpass when the conformational rearrangements are facilitated in the case of more flexible polymeric structures. Nevertheless in the semicrystalline polymer the limit state of the structural relaxation process predicted by the model simulation is quite far from equilibrium $\delta / \Delta C_{\mathrm{p}}=0.59$. The number of conformations available for the amorphous phase confined between lamellae is much smaller than in the pure amorphous chains. Thus, the collapse of mobility that stops the structural relaxation and prevents the material to reach the equilibrium state takes place in the semicrystalline polymer at a higher value of the configurational entropy than in the fully amorphous one.

From the parameters presented in Table 1 it was possible to simulate the temperature dependence of the relaxation times of NA-PLLA and SC-PLLA for a particular case-for a heating at $20{ }^{\circ} \mathrm{C} \mathrm{min}^{-1}$ after a quenching at $40{ }^{\circ} \mathrm{C} \mathrm{min}-1$. These results are shown in Fig. 5 (symbols). At low reciprocal temperatures a clear curvature is found, usually described by the well-known Vogel-Fulcher-TammannHesse (VFTH) equation. The temperature dependence of such relaxation times in equilibrium, according to the VFTH

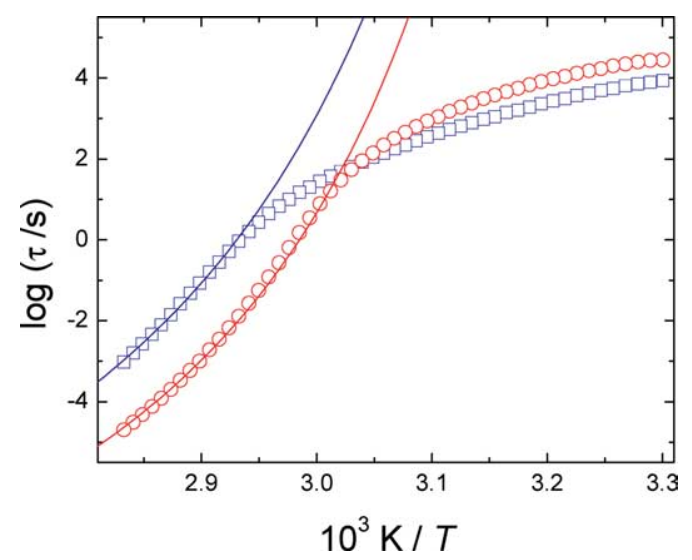

Fig. 5. Temperature dependence of the relaxation times calculated for NAPLLA (circles) and SC-PLLA (squares) during a $20^{\circ} \mathrm{C} \mathrm{min}^{-1}$ heating can, after a quenching at $40{ }^{\circ} \mathrm{C} \mathrm{min}{ }^{-1}$. The computer simulations were conducted with the sets of parameters in Table 1. The solid lines correspond to the equilibrium relaxation times, modelled by the VFTH equation. equation, is also displayed in Fig. 5 for both materials (solid lines).

In the equilibrium region the relaxation map is clearly shifted towards higher temperatures for the crystalline material, with respect with NA-PLLA. This is reflected, for example, in the glass transition temperature, that is often defined as the temperature at which $\tau=100 \mathrm{~s}$. Table 2 presents the values of $T_{\mathrm{g}}$, determined in such a way, for both materials. A difference of ca. $5{ }^{\circ} \mathrm{C}$ is detected, which would be hardly determined using the DSC reference scans, especially for the case of SC-PLLA. However, after an ageing period in the glassy state it was possible to distinguish the two $T_{\mathrm{g}} \mathrm{s}$ by DSC, and a similar difference was found between the glass transition of the bulk-like and constrained amorphous phases in PLLA [31]. The results in the equilibrium region allow to determine the apparent activation energy at $T_{\mathrm{g}}$ in equilibrium:

$\Delta h^{*}=\left.R \frac{\mathrm{d} \ln \tau^{\mathrm{eq}}}{\mathrm{d}(1 / T)}\right|_{T_{\mathrm{g}}}$

the values of $\Delta h^{*}$ are shown in Table 2, being higher for SC-PLLA. Also for the PET system, higher values of $\Delta h^{*}$ were found for the crystalline materials with respect to the amorphous polymer. This parameter may be used to obtain informations about the fragility index, related to the magnitude of the decrease of $\log \tau$ with decreasing $T_{\mathrm{g}} / T$ at $T_{\mathrm{g}}$ [44-46]:

$m=\left.\frac{\mathrm{d} \log \tau^{\mathrm{eq}}}{\mathrm{d}\left(T_{\mathrm{g}} / T\right)}\right|_{T_{\mathrm{g}}}=\frac{\Delta h^{*}}{\ln (10) R T_{\mathrm{g}}}$

The values of $m$ for the two studied materials are shown in Table 2. They are close to the ones obtained in a series of L-lactide/meso-lactide copolymers [22] $(m \sim 150)$. In that work no differences, within the experimental uncertainties, were found between the fragilities calculated in amorphous or semi-crystalline polymers. Thus, the differences in $m$ between NA-PLLA and SC-PLLA may be also not significant.

The data in Fig. 5 clearly indicate that as $T_{\mathrm{g}}$ is approaching from the high temperature region the system start to fall out of the equilibrium and a clear deviation of the previous VFT-like trend is noticed, until a quasi-Arrhenius regime is found in the glassy region. This behaviour is a consequence of the almost constant configurational entropy in the glassy state (i.e. Eq. (4) adopts an Arrhenius form). The Arrhenius plots for the two materials in the glassy state seem to be quite parallel. The slope of the simulations in Fig. 5 in the glassy state allows to calculate the apparent activation energy: 
Table 2

Glass transition features of NA-PLLA and SC-PLLA using the results obtained from the configurational entropy based model

\begin{tabular}{llccc}
\hline & $T_{\mathrm{g}}\left({ }^{\circ} \mathrm{C}\right)$ & $\Delta h^{*}\left(\mathrm{~kJ} \mathrm{~mol}^{-1}\right)$ & $m$ & $E_{\mathrm{a}}^{\mathrm{glass}}\left(\mathrm{kJ} \mathrm{mol}{ }^{-1}\right)$ \\
\hline NA-PLLA & 57.3 & 1049 & 166 & 100 \\
SC-PLLA & 62.6 & 901 & 140 & 0.10 \\
\hline
\end{tabular}

$E_{\mathrm{a}}^{\text {glass }}=R \frac{\mathrm{d} \ln \tau^{\text {glass }}}{\mathrm{d}(1 / T)}$

Very similar values of $E_{\mathrm{a}}^{\text {glass }}$ were found for NA-PLLA and SC-PLLA (Table 2). For PET it was also seen that crystallinity has no influence on this parameter [17]. This was attributed to the invariance of the configurational entropy in the glassy state with crystallinity, $S_{\mathrm{c}}^{\text {glass }}$, that should be a characteristic, nearly universal, parameter determining the temperature interval in which the glass transition takes place.

The relaxation time $\tau(t)$ in Eq. (3) is a function of both temperature and separation from equilibrium (i.e. structure). In the model proposed by Narayanaswamy [47] and then by Moynihan and his group [48], the NM model, $x$ is a parameter between 0 and 1 that defines the relative contributions of temperature and structure to the relaxation time, i.e. it can be said that defines the degree of nonlinearity of the structural relaxation process. The values of $x$ can determined by $x=E_{\mathrm{a}}^{\text {glass }} / \Delta h^{*}[47,48]$ and are also present in Table 2. No significant differences exist between the two materials, similarly to what was observed between amorphous and semi-crystalline PET [17]. The low values of $x$ reflect that, for these samples, the contribution of structure to the relaxation time is more important than the contribution of temperature $[47,48]$.

We may then conclude that crystallinity in PLLA has two main effects on the overall glass transition dynamics: A shift of the glass transition temperature to higher values and a broadening of the distribution of relaxation times. The influence of the geometrical confinement on the glass transition behaviour may be analysed under the framework of the Adam and Gibbs theory [28], using the concept of the cooperatively rearranging regions (CRR). They are defined as the smallest regions around a relaxing entity that can undergo a transition to a new configurational state without requiring simultaneous configurational changes outside its boundaries. The correlation length, $\xi$, of the CRRs assigned to the glass transition relaxation in glass formers decreases with increasing temperature. The $\xi$ value near glass transition depends on the material and, as shown in an extensive study by Hampel et al. [49], it varies typically between 1.0 and $3.5 \mathrm{~nm}$. The confinement of an amorphous phase within regions of such order of magnitude should lead to a broadening of the glass transition and a shift to higher temperatures [15,50]. These findings were interpreted by relating the size of the amorphous regions and the correlation lengths for conformational mobility. We may also include the difference found in the glass transition dynamics studied by DSC on the PLLA samples under similar arguments.
Let first suppose that for amorphous (unconstrained) PLLA the glass transition during heating takes place when the mean correlation length of the unconfined chains is below a certain value, $\xi_{\mathrm{am}}$. For SC-PLLA, during heating, the amorphous domains that are away from the crystallites (or the rigid amorphous phase) by a distance higher than $\xi_{\mathrm{am}} / 2$ will acquire mobility at temperature close to the ones for the bulk amorphous material. If the distance of the amorphous domains to the rigid walls is below $\xi_{\text {am }} / 2$ then the temperature should increase until the correlation length is below this distance and above it the domain may undergo conformational changes within the time scale of the experiment; this happens at a temperature that is higher than the glass transition temperature of the unconfined amorphous phase. This explanation seems to be compatible with a correlation found between the thickness of the amorphous mobile layer and the correlation length measured in poly (trimethylene terephthalate) crystallised at different conditions, in order to produce different lamellar morphologies [51]. In inter-lamellar regions with thicknesses higher than $\xi_{\text {am }}$ there will be a fraction of amorphous domains that will be close to the walls than $\xi_{a m} / 2$, and, in this case, a distribution of distances between the polymeric chains and the rigid walls lower than that value will exist. Therefore, it is expectable the existence of dynamic heterogeneities for the segmental mobility in semi-crystalline systems, resulting in a broader distribution of characteristic times, as compared with the bulk amorphous material.

Thus, in a dynamic point of view, the amorphous phase between the crystalline lamellae may be seen as a different phase with respect with the bulk amorphous one. However, the domains that are relaxing are composed by the same material, and differ only in average size and size distribution. This may explain the similar properties of the glassy phase (activation energy and $x$ parameter) and in fragility found in NA-PLLA and SC-PLLA.

Other arguments can explain the effect of confinement on the glass transition features. Some authors suggest that two contributions should be taken into account: The effect of geometrical confinement itself and the effect resulting from the interactions with the surface [52-54]. A discussion based on such assumption can be found elsewhere also for a PLLA system [31].

\section{Main conclusions}

Significant differences in the glass transition dynamics and structural relaxation behaviour were found between a 
nearly amorphous and a semi-crystalline PLLA (NA-PLLA and SC-PLLA) investigated by DSC. From a quantitative point of view, a phenomenological model based on the configurational entropy concept was used to describe the results and allowed to extract physical-meaningful parameters for both materials. It was found that (i) from the lower value of the KWW $\beta$ parameter in SC-PLLA, the distribution of relaxation times should be broader in SC-PLLA than in NA-PLLA; (ii) no significant differences in $B / \Delta C_{\mathrm{p}}\left(T_{\mathrm{g}}\right)$ and $T_{2}$ were found between both materials as well as on the fragility index; (iii) the $\delta$ values are small in NA-PLLA indicating that the equilibrium state below $T_{\mathrm{g}}$ could be almost reached at sufficient long times, but in the SC-PLLA the limit state of the structural relaxation process will be quite far of the equilibrium state; (iv) the glass transition, defined as the temperature at which $\tau=100 \mathrm{~s}$, is shifted to higher temperatures for the case of SC-PLLA; and (v) the main features of the glassy state (e.g. $E_{\mathrm{a}}^{\text {glass }}$ and $x$ ) are found to be almost independent on the crystallinity. The results were interpreted by assuming that the mobility of the amorphous phase confined between the crystalline lamellae is dynamically distinct from that of the bulk amorphous phase. In the former case, and under the Adam and Gibbs CRR concept, the amorphous chains that are away from the crystalline regions by a distance less than the correlation length of the bulk amorphous phase will eventually undergo conformational rearrangement at higher temperatures. Therefore, in SC-PLLA the existence of dynamically heterogeneous environments will broaden the distribution of relaxation times and shift it to higher temperatures.

\section{Acknowledgements}

Financial support for this work was provided by FCT, through the POCTI and FEDER programmes (J.F.M. and N.M.A.) and by the Spanish Science and Technology Ministry through the MAT2003-05391-C03-01 project (J.L. G.R. and M.S.S.). The authors thank Kadriye Tuzlakoglu for providing the PLLA used in this work and Maria de Fátima Alves for the help in the experimental part of the work.

\section{References}

[1] Södegard A, Stolt M. Prog Polym Sci 2002;27:1123.

[2] Thomson RC, Wake MC, Yaszemski MJ, Mikos AG. Adv Polym Sci 1995;122:245.

[3] Kim HD, Bae EH, Kwon IC, Pal RR, Nam JD, Lee DS. Biomaterials 2004;25:2319.

[4] Iannace S, Nicolais L. J Appl Polym Sci 1997;64:911.

[5] Miyata T, Masuko T. Polymer 1998;39:5515.

[6] Di Lorenzo ML. Polymer 2001;42:9441.

[7] Reeve M, McCarthy S, Downey M, Gross R. Macromolecules 1994; 27:825.

[8] Cam D, Hyon S, Ikada Y. Biomaterials 1995;16:833.
[9] MacDonald R, McCarthy S, Gross R. Macromolecules 1996;29:7356.

[10] Tsuji H, Ikada Y. Polym Degrad Stab 2000;67:179.

[11] Tsujo H, Ikada Y. Polymer 1995;36:2709.

[12] Celli A, Scandola M. Polymer 1992;33:2699.

[13] Liao K, Quan D, Lu Z. Eur Polym J 2002;38:157.

[14] Montserrat S, Cortés P. J Matter Sci 1995;30:1790.

[15] Dobbertin J, Hensel A, Schick C. J Therm Anal 1996;47:1027.

[16] Zhao J, Song R, Zhang Z, Linghu X, Zheng Z, Fan Q. Macromolecules 2001;34:343.

[17] Alves NM, Mano JF, Balaguer E, Meseguer Dueñas JM, Gómez Ribelles JL. Polymer 2002;43:4111.

[18] Ezquerra TA, Baltà-Calleja FJ, Zachmann HG. Polymer 1994;35: 2600.

[19] Fukao K, Miyamoto Y. J Non-Cryst Solids 1997;212:208.

[20] Alvarez C, Šics I, Nogales A, Denchev Z, Funari SS, Ezquerra TA. Polymer 2004;45:3953.

[21] Mijovic J, Sy J-W. Macromolecules 2002;35:6370.

[22] Kanchanasopa M, Runt J. Macromolecules 2004;37:863.

[23] Gómez Ribelles JL, Monleón Pradas M. Macromolecules 1995;28: 5867.

[24] Gómez Ribelles JL, Vidaurre Garayo A, Cowie JMG, Ferguson R, Harris S, McEwen IJ. Polymer 1998;40:183.

[25] Salmerón M, Torregrosa C, Vidaurre A, Meseguer Dueñas JM, Monleón Pradas M, Gomez Ribelles JL. Colloid Polym Sci 1999;277: 1033.

[26] Mano JF, Alves NM, Meseguer Dueñas JM, Gómez Ribelles JL. Polymer 1999;40:6545.

[27] Cameron N, Cowie JMG, Ferguson R, Gómez Ribelles JL, Más Estellés J. Eur Polym J 2002;38:597.

[28] Adam G, Gibbs JH. J Chem Phys 1965;43:139.

[29] Yavuz H, Babac C, Tuzlakoglu K, Piskin E. Polym Degrad Stab 2002; $75: 431$.

[30] Salmerón Sánchez M, Gómez Ribelles JL, Hernández Sánchez F, Mano JF. Thermochimica Acta 2005;45:92.

[31] Wang Y, Gómez Ribelles JL, Salmerón Sánchez M, Mano JF. Macromolecules 2005;38:4712.

[32] Fisher EW, Sterzel H, Wagner G. Kolloid Z Z Polym 1973;251:980.

[33] Albrecht T, Strobl G. Macromolecules 1995;28:5827.

[34] Wunderlich B. Prog Polym Sci 2003;28:383.

[35] Hu W, Albrecht T, Strobl G. Macromolecules 1999;32:7548.

[36] Mutter R, Stille W, Strobl G. J Polym Sci, Polym Phys Ed 1993;31:99.

[37] Mathot VBF. Polymer 1984;25:579.

[38] Angell CA. Polymer 1997;26:6261.

[39] van Danne H, Fripiat JJ. J Chem Phys 1978;62:3365.

[40] Cohen MH, Turnbull D. J Chem Phys 1959;31:1164.

[41] Cohen MH, Grest GS. Phys Rev B 1979;20:1077. Cohen MH, Grest GS. Adv Chem Phys 1981;48:370.

[42] Saiter A, Oliver JM, Saiter JM, Gómez Ribelles JL. Polymer 2004;45: 2743.

[43] Ren J, Urakawa O, Adachi K. Macromolecules 2003;36:210.

[44] Angell CA. J Non-Cryst Solids 1991;131-133:13.

[45] Angell CA. Science 1995;267:1924.

[46] Ediger MD, Angell CA, Nagel SR. J Chem Phys 1996;100:13200.

[47] Narayanaswamy OS. J Am Chem Soc 1971;54:491.

[48] Moynihan CT, Macedo PB, Montrose CJ, Gupta PK, DeBolt MA, Dill JF, et al. Ann NY Acad Sci 1976;279:15.

[49] Hempel E, Hempel G, Hensel A, Shick C, Donth E. J Phys Chem 2000;B104:2460.

[50] Sappelt D, Jäckle J. J Phys A: Math Gen 1993;26:7325.

[51] Hong P-D, Chuang W-T, Yeh W-J, Lin T-L. Polymer 2002;43:6879.

[52] Schönhals A, Goering H, Brzezinka KW, Schick C. J Phys: Condens Matter 2003;15:S1139.

[53] Kremer F, Hartmann L, Serghei A, Pouret P, Léger L. Eur Phys J E 2003; $12: 139$.

[54] Schönhals A, Goering H, Schick C, Frick B, Zorn R. Colloid Polym Sci 2004;282:882. 